\title{
Robust Visualization of the Dental Occlusion by a Double Scan Procedure ${ }^{\star}$
}

\author{
Filip Schutyser ${ }^{1}$, Gwen Swennen ${ }^{2}$, and Paul Suetens ${ }^{1}$ \\ 1 Department of Medical Image Computing (Radiology - ESAT/PSI), \\ Faculties of Medicine and Engineering, University Hospital Gasthuisberg, \\ Katholieke Universiteit Leuven, BE \\ filip.schutyser@uz.kuleuven.ac. be \\ 2 Department of Plastic Surgery, University Hospital Brugmann, \\ Université Libre de Bruxelles, BE
}

\begin{abstract}
A detailed visualization of the dental occlusion in 3D imagebased planning environments for oral and maxillofacial planning is important. With CT imaging however, this occlusion is often deteriorated by streak artifacts caused by amalgam fillings. Moreover, more detailed surface information at the level of the dental cuspids is often desired.

In this paper, a double scan technique is introduced to image the dental occlusion by means of a newly designed $3 \mathrm{D}$ splint. The patient wears this splint between the upper and lower teeth during CT-scan. In a second step, the splint is positioned between the plaster casts of the upper and lower jaw, and this setup is scanned. Based on markers in the 3D splint, both data sets are fused and a combined visualization is possible. The accuracy, robustness and applicability in clinical routine is shown.

This technology enables meticulous 3D cephalometric analysis, detailed maxillofacial planning and opens possibilities towards intraoperative support.
\end{abstract}

\section{Introduction}

In 3D image-based planning environments for oral and maxillofacial surgery 1, it is important to inspect the dental occlusion carefully. For total and partial edentulous patients, it is important to co-visualize the denture with the bone. In this way, oral implant planning is not only driven by bone related issues, but also by prosthetics. This improves the esthetic result. For patients with a complete dentition facing orthognatic surgery, the surgical planning implies optimizing the occlusion. Therefore, an accurate visualization of the occlusion is important.

To visualize bony structures of the head, CT (computerized tomography) is the preferred modality. However, some important drawbacks are identified: amalgam fillings generate important streak artifacts, and the acrylic resin that is used to make dentures, shows the same Hounsfield units as soft tissues.

\footnotetext{
* This work partly belongs to the Flemish government IWT GBOU 020195 project on Realistic image-based facial modelling for forensic reconstruction and surgery simulation and the IWT-040310 project on fusion of medical CT image volumes with digitizations of synthetic models.
} 
In the field of oral implant planning, we introduced the double scan procedure 2. With this CT protocol, the patient wearing a denture equipped with gutta percha markers is CT-scanned in a first step. Then, the prosthesis alone is CT-scanned. From this scan, the prosthesis is robustly segmented. Based on the markers, visible in both scans, the prosthesis is rigidly registered towards the scan of the patient.

Nkenke et al. 3] add a digitization of plaster models, acquired by optical surface scanning or CT imaging, to the patient's CT image volume by registration of surfaces of teeth. They show that the accuracy of this method strongly reduces with the presence of artifacts.

Gateno et al. 4] explain an in vitro test of the fusion of a laser scanned dental impression with a CT scan of a patient wearing this impression. Some extra characteristic shapes are attached to the impression. Based on these shapes, the registration is performed. However, as such, this technique is not applicable in clinical routine. No patient examples are shown. Moreover, special equipment is needed for the laser scan.

In this paper, we show that the double scan procedure can be extended to robustly image the dental occlusion of patients with a full dentition with a high detail. The registration was performed fully automatically. The method has been validated on 10 cadaver skulls, and 7 patients.

\section{Method}

\subsection{D Splint}

During orthodontic treatment, and for diagnostic work-up of orthognatic surgery, plaster casts are routinely made. These plaster casts are an accurate copy of the actual dentition of the patient. To record the relative position of the plaster casts of the dental arches, a splint is produced.

During CT-scan, the patient gently bites on this splint. In a second scan, the plaster models with that splint are CT-scanned. If a rigid registration of the splint in both scans is possible, the CT-scan of the plaster models is fused with the patient scan.

For this purpose, the splint is modified. Spherical gutta percha markers are added to the splint. This causes bright spots on the CT images of the patient and the plaster models. This will be the basis for the matching procedure. There is a trade-off for the size of the markers. To make them easily detectable, rather large balls are preferred. However, larger balls generate also CT artifacts. A diameter of $1.5 \mathrm{~mm}$ is found to be optimal after extensive tests.

Since the splint has an almost planar structure, an extension perpendicular to the occlusal plane is added to improve the registration accuracy. To have a comfortable solution for the patient, this extension is mounted on a bar leaving the mouth. In this way, the natural shape of the face is not disturbed.

This 3D splint (see figure 1) is the key to obtain an accurate visualization of the occlusion. This 3D splint is produced with a hard, radiolucent acrylic resin which is not toxic. It can be easily produced and it is cheap. 


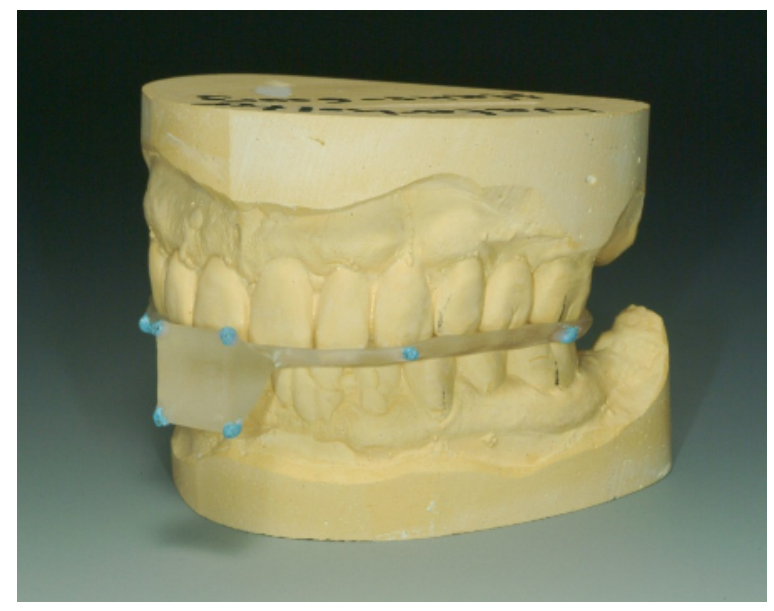

Fig. 1. The 3D splint is equipped with gutta percha markers and has an extraoral extension. The patient wears this splint during CT-scan. The splint is also scanned together with the plaster models.

\subsection{Registration}

Gutta percha is very radio-opaque. Therefore, candidate markers are extracted by thresholding. To classify these candidates into markers and other structures, two measures are computed:

1. The Normalised Shape Index (NSI), defined in equation 1, expresses the resemblance of an object to sphere. $k$ is defined so that NSI $=1$ for a sphere. NSI must be smaller then a threshold value to be a marker.

$$
\mathrm{NSI}=k \frac{\text { volume }}{\text { surface }}
$$

2. The volume of the object needs to be larger then a threshold value to exclude small structures due to noise or artifacts.

To find the rigid registration matrix based on the centers of the markers in both image volumes, the correspondence between both sets is needed. Given the sparse set of points, algorithms like Iterative Closest Points (ICP) [5] often fail. Since we aim for a fully automatic procedure, a dedicated approach is needed.

The Euclidean distance between two markers of set 1, equals the distance between two markers of set 2 . This holds for all distances between possible marker pairs. If markers $a$ and $b$ correspond with markers $a^{\prime}$ and $b^{\prime}$, then is marker $c^{\prime}$ a corresponding marker to $c$ if $\|a c\|-\left\|a^{\prime} c^{\prime}\right\|<$ threshold, and $\|b c\|-\left\|b^{\prime} c^{\prime}\right\|<$ threshold. Since an initial set of corresponding points is not known, the algorithm is applied for all candidate correspondences, and the solution with the largest set of corresponding markers is kept.

The translation and rotation matrix are finally computed using a least squares approach [6]. 


\section{Results}

This method was tested and validated on a set of 10 cadaver skulls. For each skull, a 3D splint and plaster models were produced. The skulls and plaster models were CT-scanned (GE LightSpeed QX/i, 120 kV, 100 mAs, spiral CT). In order to reduce the number of axial slices affected by streak artifacts, the occlusal plane was positioned perpendicular on the CT-table. For the models, the smallest available slice thickness was selected and the axial slices were reconstructed with a bone kernel and voxel size of $(0.3,0.3,0.3) \mathrm{mm}$. The patients were scanned with a lower resolution, to reduce the radiation dose, and the axial slices were reconstructed with a bone kernel and voxel size of $(0.35,0.35,1.0) \mathrm{mm}$.

The registration results are listed in table 1 A mean error of $0.14 \mathrm{~mm}$ is a fairly acceptable accuracy for the application of 3D image-based planning given

Table 1. This table lists the registration results for 10 cadaver skulls

\begin{tabular}{c|c|c|c|c|c} 
Skull & Nr markers & $\begin{array}{c}\text { Mean error } \\
(\mathrm{mm})\end{array}$ & $\begin{array}{c}\text { Standard deviation } \\
(\mathrm{mm})\end{array}$ & $\begin{array}{c}\text { Max. error } \\
(\mathrm{mm})\end{array}$ & $\begin{array}{c}\text { Min. error } \\
(\mathrm{mm})\end{array}$ \\
\hline 1 & 10 & 0.13 & 0.05 & 0.19 & 0.08 \\
2 & 9 & 0.14 & 0.04 & 0.17 & 0.09 \\
3 & 10 & 0.14 & 0.07 & 0.28 & 0.03 \\
4 & 9 & 0.17 & 0.07 & 0.25 & 0.08 \\
5 & 9 & 0.12 & 0.03 & 0.16 & 0.06 \\
6 & 11 & 0.18 & 0.06 & 0.30 & 0.10 \\
7 & 8 & 0.11 & 0.04 & 0.20 & 0.07 \\
8 & 11 & 0.08 & 0.03 & 0.13 & 0.03 \\
9 & 8 & 0.12 & 0.04 & 0.21 & 0.08 \\
10 & 12 & 0.18 & 0.08 & 0.35 & 0.07 \\
\hline
\end{tabular}

Overall mean error

$0.14 \mathrm{~mm}$

Overall standard deviation of error $0.03 \mathrm{~mm}$

Table 2. This table lists the registration results for 7 patients

\begin{tabular}{c|c|c|c|c|c} 
Skull & Nr markers & $\begin{array}{c}\text { Mean error } \\
(\mathrm{mm})\end{array}$ & $\begin{array}{c}\text { Standard deviation } \\
(\mathrm{mm})\end{array}$ & $\begin{array}{c}\text { Max error } \\
(\mathrm{mm})\end{array}$ & $\begin{array}{c}\text { Min error } \\
(\mathrm{mm})\end{array}$ \\
\hline 1 & 5 & 0.13 & 0.04 & 0.19 & 0.09 \\
2 & 6 & 0.17 & 0.06 & 0.28 & 0.09 \\
3 & 4 & 0.15 & 0.07 & 0.24 & 0.10 \\
4 & 4 & 0.15 & 0.04 & 0.20 & 0.11 \\
5 & 8 & 0.16 & 0.05 & 0.24 & 0.10 \\
6 & 9 & 0.21 & 0.10 & 0.41 & 0.09 \\
7 & 14 & 0.15 & 0.07 & 0.34 & 0.04 \\
\hline
\end{tabular}

Overall mean error 


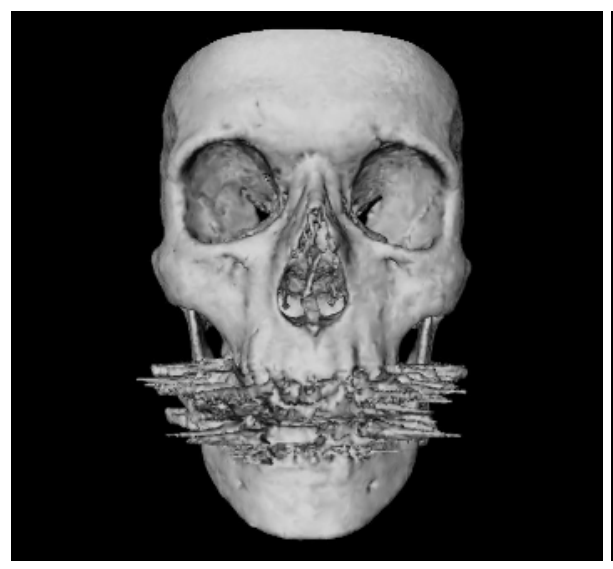

(a)

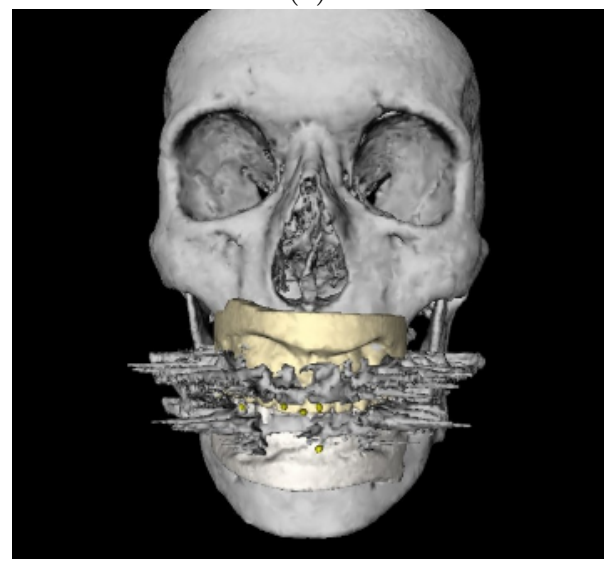

(c)

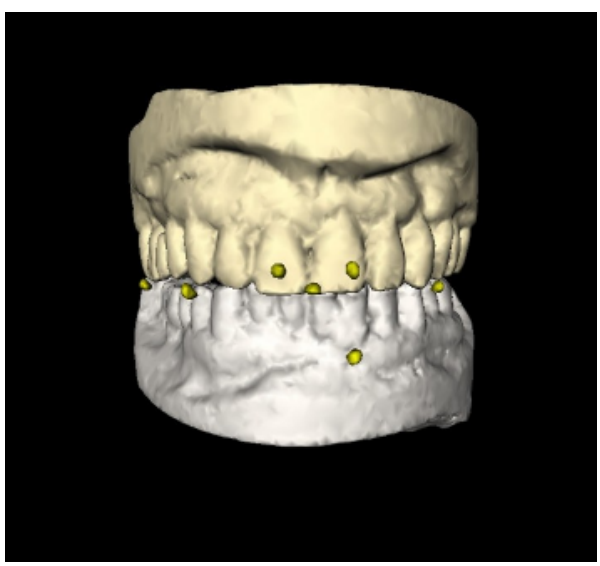

(b)

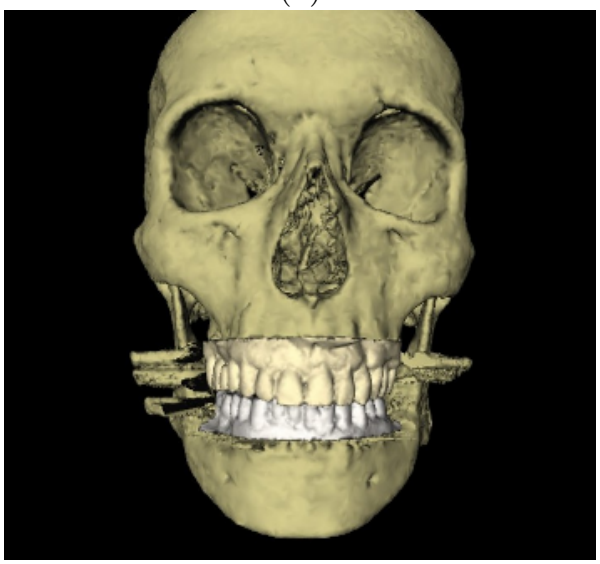

(d)

Fig. 2. This figure shows how the registration with the $3 \mathrm{D}$ splint enables detailed imaging of the occlusion. Streak artifacts corrupt CT images (a). Plaster models can be accurately imaged with CT-imaging (b). Based on the 3D-splint, both data sets can be fused (c) and the surface description extracted from the plaster model scan replaces the corrupted data $(d)$.

the resolution of the $\mathrm{CT}$ data sets. Therefore, this technique was applied on patients with amalgam fillings or orthodontic brackets with steel wires.

Seven patients with severe amalgam fillings were selected for this test. All of them received a 3D-splint, and the double-scan technique is applied. An excessive amount of gutta percha markers is inserted in the splint because some of them will be submerged in the artifacts. All registrations are performed fully automatically with a mean registration error of $0.16 \mathrm{~mm}$ as shown in table 2 .

The registered surfaces are co-visualized and the teeth surface from the plaster models replaces the teeth surface from the patient scan (see figure2). In this way, a very detailed dental occlusion is visualized. 


\section{Discussion}

The double scan procedure is a protocol that enables robust visualization of the dental occlusion. First the patient is scanned with an adapted material at the level of the occlusion. Then this adapted material is scanned alone.

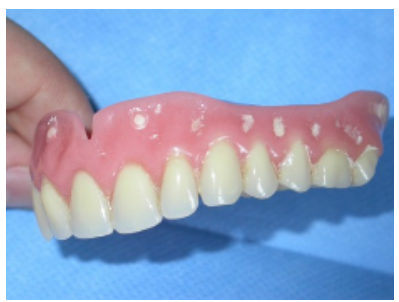

(a)

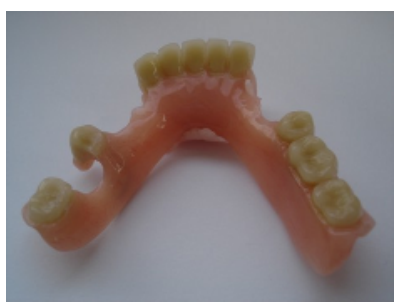

(b)

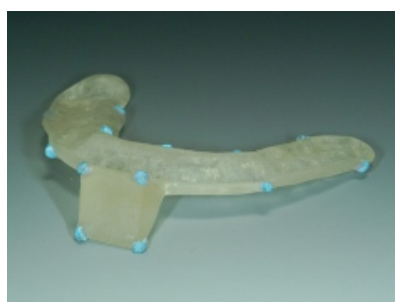

(c)

Fig. 3. This figure illustrates the evolution of a denture for a complete edentulous patient (a), over a partial denture (b) towards the 3D splint (c). Based on these materials, an accurate visualization of the dental occlusion becomes possible.

When the patient is edentulous, the prosthesis equipped with gutta percha markers is used to visualize the occlusion. For partial edentulous patients, a partial prosthesis, equipped with gutta percha markers and, if needed, support on the neighboring teeth is applied. A bite index is applied to secure the correct occlusion during CT-scan. For dentate patients, the "prothesis" reduces to the $3 \mathrm{D}$ splint. This is illustrated in figure 3 .

Some salient features of this approach can be enumerated:

1. The second scan can be performed with a very high resolution since the patient is not involved and thus radiation dose is not relevant.

2. Since the patient gently bites on the 3D splint, the referring clinician knows that the occlusion during CT-scan is correct. In current clinical practice, this is not guaranteed because the patient is laying on the CT table in stead of sitting on a chair and the patient might be not at ease for the CT exam.

3. Although the patient scan might have excessive streak artifacts, the second scan lacks artifacts. By this method, the clinical hindrance caused by those artifacts is reduced.

4. The markers are robustly detected and the scans are accurately registered as shown in the results.

5. This double scan technique is easy to implement in daily clinical routine.

6. It is a cheap procedure. The cost of inserting gutta percha markers and making the $3 \mathrm{D}$ splint is almost negligible.

This technology has several applications in the field of orthodontics, maxillofacial surgery and plastic surgery. Detailed visualization of the dental occlusion allows accurate 3D cephalometric analysis including landmarks located 
on the dental occlusion. Surgical optimization of the dental occlusion can be meticulously planned, and intraoperative surgical support to obtain the planned solution can be derived.

\section{Conclusion}

The double scan procedure is a robust and accurate method to visualize the dental occlusion. Since the information needed to visualize the occlusion is acquired in a second scan without the patient, a highly detailed artifact free scan can be obtained. Through the use of a large number of gutta percha points, the registration is shown to be accurate and fully automatic.

This technology is useful for detailed 3D cephalometric analysis, accurate maxillofacial surgery planning and intra-operative support.

\section{References}

1. Schutyser, F., Van Cleynenbreugel, J., Ferrant, M., Schoenaers, J., Suetens, P.: Image-based 3d planning of maxillofacial distraction procedures including soft tissue implications. (2000) 999-1007

2. Verstreken, K., Van Cleynenbreugel, J., Martens, K., Marchal, G., van Steenberghe, D., Suetens, P.: An image-guided planning system for endosseous oral implants. IEEE Transactions on Medical Imaging 17 (1998) 842-852

3. Nkenke, E., Zachow, S., Benz, M., Maier, T., Veit, K., Kramer, M., Benz, S., Häusler, G., Neukam, F.W., Lell, M.: Fusion of computed tomography data and optical 3d images of the dentition for streak artefact correction in the simulation of orthognathic surgery. Dentomaxillofacial Radiology 33 (2004) 226-232

4. Gateno, J., Xia, J., Teichgraber, J.F., Rosen, A.: A new technique for the creation of a computerized composite skull model. Journal of Oral and Maxillofacial Surgery 61 (2003) 222-227

5. Besl, P., McKay, N.: A method for registration of 3-d shapes. IEEE Transaction on Pattern Analysis and Machine Intelligence 14 (1992) 239-256

6. Arun, K., Huang, T., Blostein, S.: Least-squares fitting of two 3-d point sets. IEEE Transaction on Pattern Analysis and Machine Intelligence 9 (1987) 698-700 